\section{Sipuleucel-T induces humoral antigen spread in patients with mCRPC}

New research reveals that treatment with sipuleucel-T results in increased levels of several self-antigens, and a concomitant increase in IgG responses, known as antigen spread. These findings might explain the improved overall survival (OS) of patients with metastatic castrationresistant prostate cancer ( $\mathrm{mCRPC}$ ) treated with sipuleucel-T versus placebo as part of the IMPACT trial, published in 2010.

Serum samples from 142 patients with mCRPC who received treatment with sipuleucel-T as part of the IMPACT trial were analysed using protein microarrays. Patients treated with sipuleucel-T had a $\geq 2$-fold increase in serum IgG levels post-treatment compared with pretreatment. At 10-weeks after treatment, significant increases in the presence of a range of secondary antigens were detected, including PSA and antigens not previously associated with mCRPC such as LGALS3 (lectin, galactoside-binding, soluble, 3 ).
Patients receiving sipuleucel-T who had an anti-PSA IgG response also had significantly better OS than patients receiving placebo; however, patients receiving sipuleucel-T who had no detectable anti-PSA IgG response had no significant differences in OS compared with placebo. A similar effect was observed in patients with or without an anti-LGALS3 IgG response.

Patients with IgG responses to a greater number of antigens also had more favourable outcomes, and this effect became more conspicuous as the number of IgG responses increased. When earlier ( 2 weeks post-treatment) or later (22 weeks post-treatment) timepoints were investigated, similar IgG response were detected, suggesting that the response to sipuleucel is both rapid and sustained.

Taken together, these findings suggest explain the encouraging therapeutic that sustained antigen spread might

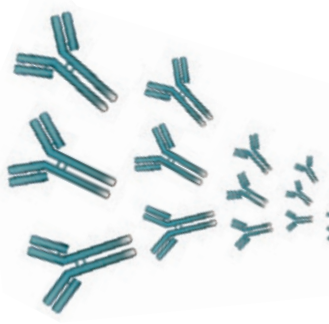

effects of sipuleucel-T in patients with mCRPC. Only a limited

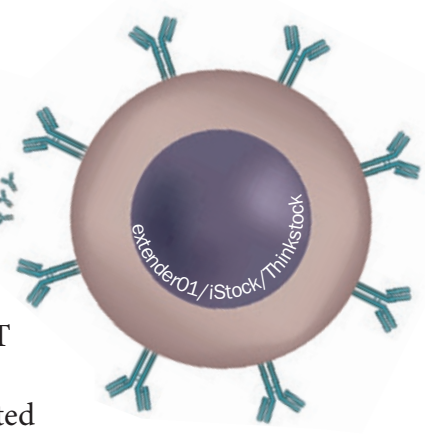
number of IgG responses to sipuleucel-T were investigated in this study, therefore, future studies that investigate a greater number of potential IgG responses might better capture the full spectrum of sipuleucel-T induced alterations. Treatment-induced responses to secondary antigens might also reveal future therapuetic targets.

Peter Sidaway

Original article GuhaThakurta, D. et al. Humoral immune response against non-targeted tumor antigens after treatment with sipuleucel-T and its association with improved clinical outcome. Clin. Cancer Res. doi:10.1158/1078-0432.CCR-14-2334 